\title{
Digital Radio over Fiber Distribution using Millimetre Wave Bridging
}

\author{
Haymen Shams ${ }^{*}$, Tongyun Li ${ }^{2}$, Cyril C. Renaud ${ }^{1}$, Alwyn J. Seeds ${ }^{1}$, Richard Penty ${ }^{2}$, Martyn Fice ${ }^{1}$, Ian White ${ }^{2}$ \\ ${ }^{l}$ Department of Electronic and Electrical Engineering, University College London, London, U.K \\ ${ }^{2}$ Centre for Photonic Systems, Electrical Division, Department of Engineering, University of Cambridge, Cambridge, U.K \\ *h.shams@ucl.ac.uk \\ Abstract: This paper demonstrates a novel digital radio-over-fiber system using a millimeter- \\ wave bridge giving access where fiber cannot. The system can transport multiple digitised LTE- \\ compatible signals with high spectral efficiency, low latency, and wide dynamic range. \\ OCIS codes: (060.5625) Radio frequency photonics; (350.4010) Microwave; (060.4510) Optical communications
}

\section{Introduction}

Radio over fibre (RoF) based wireless fronthaul is well established for delivering multiple streams of wireless carriers in a service agnostic and neutral host manner [1,2]. Currently, these systems have been demonstrated for carrier frequencies up to $20 \mathrm{GHz}$ [3], but with increasing mobile capacity demand, there is interest in the use of millimetre-wave $(\mathrm{mmW})$ bands, between 30 and $300 \mathrm{GHz}$, where these frequencies can easily support 200 times greater capacity than the traditional cellular spectrum [3]. Both analogue and digital techniques can be used for signal distribution, analogue radio transmission being very flexible but suffers from limited channel number capacity, noise, and nonlinear impairments as well as being difficult to integrate with existing digital network infrastructure. As a result, industrial solutions using digital RoF (D-RoF) have been developed for last-mile wireless coverage. The common public radio interface (CPRI) is the most widely used digital interface connecting baseband unit (BBU) and remote radio head (RRH) in a centralised radio access network (C-RAN) architecture. The line rate of CPRI ranges from 614.4 Mbps to $24.33 \mathrm{Gbps}$ depending on the number of carriers and antennas [4]. The traditional approach has been to carry the high-speed CPRI data over optical fibre for distribution but recent commercial products have been made available to carry CPRI over mmW to places where fibre is not available, such as Huawei's RTN 380H, Ericson's fronthaul 6392, EBlink's FrontLink and Cablefree's CPRI mmW. The key limitation of these solutions is the low spectral efficiency - a single $20 \mathrm{MHz}$ LTE band requires a $1.25 \mathrm{Gbps}$ rate to transport.

A data compression algorithm performed using field programmable gate arrays (FPGA) has been demonstrated, in which a digitised $20 \mathrm{MHz}$ LTE signal is compressed to only $400 \mathrm{Mbps}$ [5]. This solution enables an efficient system for delivering multi-band multi-carrier services for in-building wireless applications and has been shown to deliver several 2G, 3G and 4G services using packets transmitted over a single 10 Gbps network. As it is a digital transmission system, the system performance mirrors that of conventional data services and link lengths can readily be extended. In this paper, we extend the system architecture by using a $\mathrm{mmW}$ wireless link to replace optical fibre. This is expected to significantly improve the cost effectiveness of such systems in locations where fibre is unavailable or difficult to install.

\section{System Proposal}

The proposed system architecture is shown in Fig.1. In the down link, RF sources are connected to a D-RoF access unit (AU) where RF-IF conversion, digitisation and data compression take place. The low bit rate digital data is then transmitted through an optical fibre to a mmW transceiver (TRx) for upconverting to the W- band at $100 \mathrm{GHz}$. After wireless transmission, the received signal is down converted and recovered by a mmW detection unit and subsequently sent to a D-RoF remote unit (RU) for wireless distribution. The reverse approach is used for the uplink, these sending RF signals from the RU to the RF service sources. The system can also accept CPRI inputs from the BBU pool directly and perform compression on CPRI. However, CPRI is not yet an open protocol for neutralhost RF distribution. For this reason, we will only focus on direct RF input in this paper.

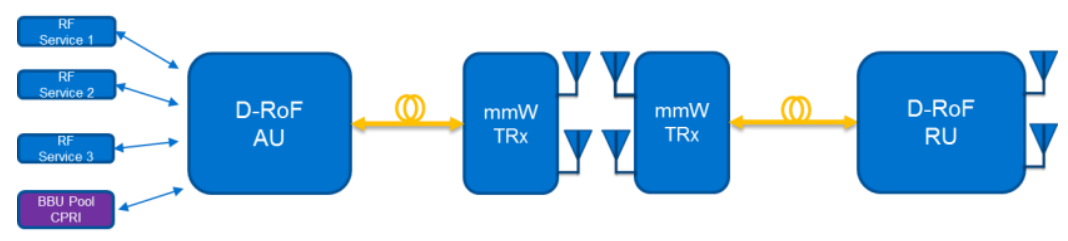

Fig.1. The proposed system architecture for the digital RF signal over mmW 


\section{Experimental Setup and Results}

The experimental system is composed of two parts: the D-RoF system and the optical to mmW convertor. In the DRoF part, the input RF signal is converted to optical pulse streams and then to $\mathrm{mmW}$, and the received optical pulses from $\mathrm{mmW} \mathrm{Rx}$ are converted back to an electrical RF signal, as illustrated in Fig. 2. The input RF signal is generated from a Rhode and Schwarz (R\&S) SMW200A signal generator. By way of example, the experiment has used an LTE-compatible signal at $37.5 \mathrm{MHz}$ IF carrier frequency with a bandwidth of $15.36 \mathrm{MHz}$. The electrical spectrum of the generated signal is shown as inset (i) in Fig. 3. A 14-bit analogue-to-digital converter/digital-toanalogue converter (ADC/DAC) pair operating at $150 \mathrm{Msps}$ is used to digitise the incoming RF signal or generate an analogue signal. The ADC/DAC is interfaced with an Altera's Stratix IV FPGA board. The FPGA board is programmed to perform the digital signal processing (DSP) algorithms including digital up/down conversion (DDC/DUC), data compression/decompression and packetisation/de-packetisation, as presented in algorithm blocks in Fig. 2. The FPGA output data line rate is at $1.25 \mathrm{Gbps}$ so that $3 \times 20 \mathrm{M}$ LTE streams are able to be accommodated. In this experiment, we replicate the compressed IQ (400Mbps) components before transmitting them to simulate a multi-service scenario. A transceiver interface card is connected to the FPGA to interface it with a commercial optical transceiver module. A small form factor plus (SFP+) optical module with a distributed feedback laser (DFB) at a wavelength of $1538.92 \mathrm{~nm}$ is used as an optical transceiver to/from the optical $\mathrm{mmW}$ generation system.

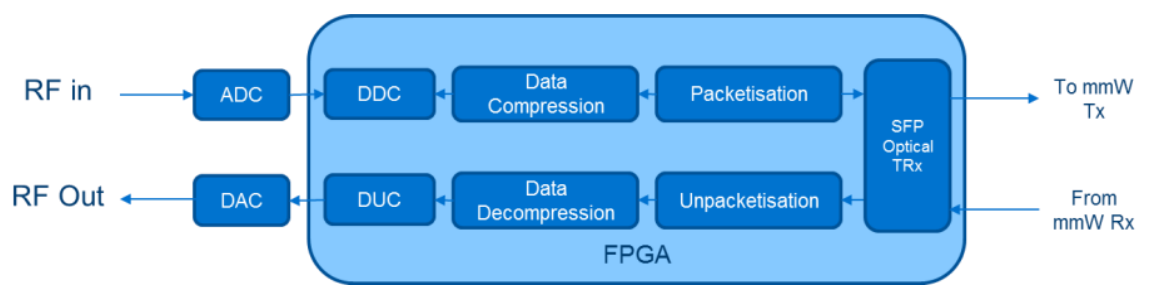

Fig. 2. Block diagram of the D-RoF test bed.

Fig. 3 shows the experimental arrangement of the proposed $\mathrm{mmW}$ bridged digital RoF system. The optical output is intensity modulated using the on-off keying data stream of the digitised RF signal and has an output power of $1.5 \mathrm{dBm}$. This modulated optical signal is transmitted over $40 \mathrm{~km}$ single mode fibre with an optical loss of $8 \mathrm{~dB}$, and then heterodyned with an external cavity laser (ECL) at $1539.71 \mathrm{~nm}$, offset by $100 \mathrm{GHz}$ from the modulated optical signal, as shown in the optical spectrum in the inset (ii) of Fig. 3. This optical signal is amplified by an erbium doped amplifier (EDFA) and filtered by an optical bandpass filter (OBPF) to remove amplified spontaneous emission. This optical signal is converted to an electrical $\mathrm{mmW}$ signal at $100 \mathrm{GHz}$ using an uni-travelling carrier photodiode (UTC-PD). The UTC-PD was an unpackaged device with a coplanar waveguide (CPW) contact. The power of the generated mmW signal is highly dependent on the fibre coupling and CPW probe contact. Wireless transmission is performed over less than $1 \mathrm{~m}$ wireless distance using a pair of directive horn antennas with $20 \mathrm{~dB}$ gain. It should be mentioned here that the wireless distance can be further extended until complete loss of the detection signal. At the $\mathrm{mmW}$ receiver, the received $\mathrm{mmW}$ signal is down converted to the IF by mixing it with a 42.4 GHz electrical LO signal in a second harmonic mixer (SHM). Then, the IF signal is down-converted to the baseband by using an envelope detector. The envelope detector removes the laser frequency fluctuation by

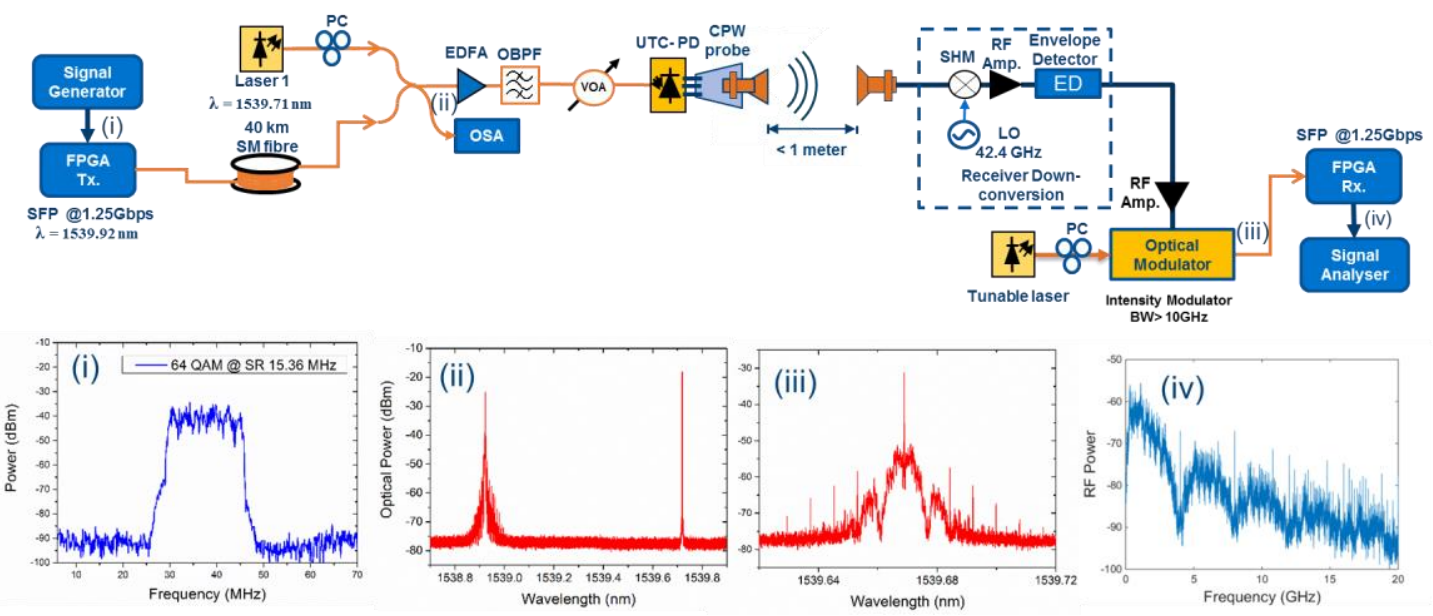

Fig. 3. Experimental arrangements for the optical $\mathrm{mmW}$ generation and detection. 
performing square law detection. To simplify this arrangement, a mmW envelope detector could be used after the horn antenna without the need of the first down-conversion process at the cost of reduced receiver sensitivity. The baseband signal is then modulated onto the optical carrier by intensity modulating a tunable laser. Insets (iii) and (iv) in Fig. 3 show the modulated optical spectrum, and the electrical spectrum of the received baseband signal. The optical signal is then received by the same optical transceiver SFP module used in the optical transmitter, and converted to electrical RF signal for evaluation by the signal analyser. The system performance was evaluated by using root mean square of error vector measurements (EVM), and the dynamic range was also obtained for three different modulation formats.

The EVM is plotted in Fig. 4 (a) at various input optical powers by measuring the received photocurrent squared in the UTC-PD, since the square of the photocurrent is directly proportional to the radiated RF power from the horn antenna, and an indicator of the amount of the injected optical power. The EVM values are maintained at less than 6\% for QPSK, 16 QAM, and 64 QAM for the photocurrents above $1 \mathrm{~mA}$, while below this it starts to increase till the signal is no longer detected. This means the wireless link can be extended for over $2 \mathrm{~km}$ distance without signal degradation, which corresponds to $8.5 \mathrm{~dB}$ free space loss. The constellation diagrams for the three modulation formats are shown in Fig. 4 (b) at the highest and lowest EVM values. The EVM measurements for different input RF powers are also determined by varying the driving electrical signal into ADC board. The EVM requirement specified by 3GPP LTE specification is $17.5 \%$ for QPSK, $12.5 \%$ for 16 QAM and $8 \%$ for 64 QAM. As shown in Fig. 4 (c), the total dynamic range of the system is between $40 \mathrm{~dB}$ and $50 \mathrm{~dB}$. With the aid of a frontend automatic gain controller (AGC), the dynamic range can further extend to over $70 \mathrm{~dB}$. These results show higher performance for dynamic range than those obtained by analog mmW [6]. Fig. 4 (d) shows the measured EVM for the received optical signal for 64 QAM after DAC. The obtained EVM values are kept constant below $6 \%$ for received optical powers above $-27 \mathrm{dBm}$. The total latency introduced in the system was $2.1 \mu \mathrm{sec}$ measured only for the FPGA processing and $\mathrm{mmW}$ wireless transmission by identifying the time offset between the input sample points and received sample points on Altera's SignalTap II waveform analyser software. These values indicate the system robustness and capability of meeting some of the most stringent requirement specified by mobile operators and neutral hosts for a real-life rollout.

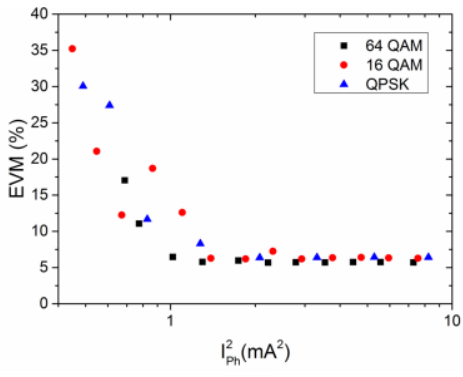

(a)

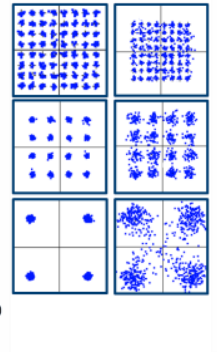

(b)

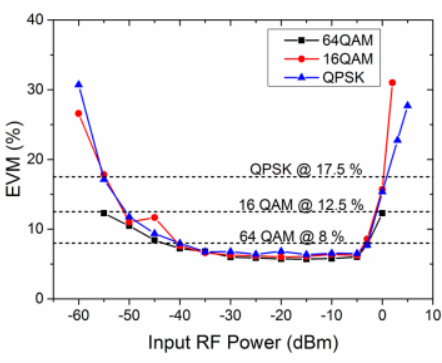

(c)

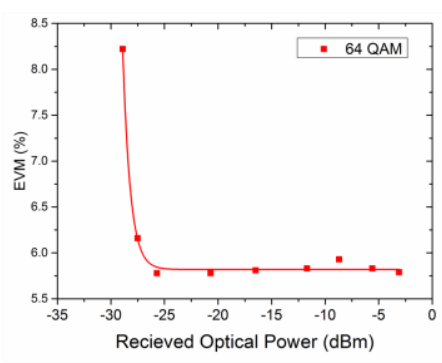

(d)

Fig. 4. (a) Measured EVM versus the received optical power, (b) constellation diagrams for QPSK, 16 QAM, and 64 QAM signal and their (c) EVM measurement versus RF input power, and (c) EVM versus the received optical power to the FPGA Rx.

\section{Conclusions}

This paper reports a system where compressed D-RoF signals are transmitted over a $100 \mathrm{GHz} \mathrm{mmW}$ wireless link, showing 3-times higher transmission efficiency than CPRI and no degradation of RF performance. The digitised RF transmission of our proposed system shows higher dynamic range than analogue $\mathrm{mmW}$. The system architecture is scalable and can support neutral host scenarios when multiple wireless carriers can be carried over a single infrastructure.

\section{Acknowledgement}

This work was funded by the UK Engineering and Physical Sciences Research Council grants for Converged Optical \& Wireless Access Networks (COALESCE) EP/P003990/1, and Coherent Terahertz Systems (COTS), $\mathrm{EP} / \mathrm{J} 017671 / 1$.

\section{References}

[1] M. J. Crisp, S. Li, A. Watts, R. V. Penty, and I. H. White, "Uplink and downlink coverage improvements of 802.11g signals using a distributed antenna network," J. Light. Technol. 25, 3388-3395 (2007).

[2] T. Li, M. Crisp, R. Penty, and I. White, "Low bit rate digital radio over fibre system," Int. Trop. Meet. Microw. Photonics 2009 1-4 (2009).

[3] P. Hartmann, et. al, "1-20 GHz Directly Modulated Radio over MMF Link," 2005 Int. Top. Meet. Microw. Photonics 44, 3-6 (2005).

[4] Common Public Radio Interface, "CPRI Specification V7.0," Stand. Doc. Specif. 0, 128 (2015).

[5] T. Li, R. V. Penty, and I. H. White, "Novel digital radio over fibre for 4G-LTE," IEEE Int. Conf. Commun. Work. ICCW $312-317$ (2015).

[6] P. T. Dat, A. Kanno, K. Inagaki, N. Yamamoto, and T. Kawanishi, "High-Spectral Efficiency Millimeter Wave-over-Fiber System for Future Mobile Fronthaul," in ECOC 2013 (2015), pp. 1-3. 